\title{
The Impact of Vaccines in Low- and High-Income Countries
}

\author{
Leif Gothefors \\ Department of Clinical Sciences/Paediatrics, Umeå University, Umeå, Sweden
}

\section{Key Words}

Vaccines $\cdot$ Immunization, expanded programme

\begin{abstract}
Vaccination has become the most effective public health measure for the control of infectious diseases after the provision of clean drinking water. The history of vaccination is marked with great hopes and some disappointments. In particular, the second half of the 20th century witnessed the development of remarkable vaccination projects. There is a possibility that polio and measles may be eradicated within a few years, but almost 3 million people - usually children $<5$ years of age - die each year from diseases that are preventable by vaccines. Developing countries are struggling to get the vaccines to children who desperately need them. However, in Europe and North America, people have become complacent about vaccines: 'these diseases are no longer a threat and the vaccine is more dangerous than the disease'. Those misconceptions have caused outbreaks of measles, diphtheria and pertussis. The international community must continue to devote the necessary resources, money and manpower to fully exploit the promise that vaccines hold for the relief of human misery.
\end{abstract}

Copyright $\odot 2008$ Nestec Ltd., Vevey/S. Karger AG, Basel

In 2007, the British Medical Journal asked their readers to nominate the most important medical advances since 1840 [1]. This not too scientific poll resulted in a top quartet: sanitation (clean water and sewage disposal), antibi- otics, anaesthesia and vaccines. If one should base one's vote on the number of lives saved, vaccines seem hard to beat (table 1).

The successful eradication of smallpox in 1977 lead to an understandable optimism that childhood diseases could also be eradicated by vaccination. In 1974, the WHO created the Expanded Programme on Immunization (EPI) to provide basic vaccines for the world's children. At that time, less than $5 \%$ of children were immunized against the 6 target diseases (tuberculosis, diphtheria, tetanus, pertussis, polio and measles) and immunization programmes were largely limited to industrialized countries, and even then, only partially implemented. Despite the intentions of the WHO (through the work of the EPI) and the good availability of cheap, safe and effective vaccines, no other pathogen has yet been eradicated on a global scale. However, the eradication of polio and measles seems to be a possibility, and the major part of this article will thus be devoted to those efforts. Only vaccines used in the EPI (now or in the near future) are dealt with.

\section{Some Definitions}

Eradication is a permanent world-wide reduction down to zero of an infection caused by a specific agent: intervention measures can thus be discontinued.

Elimination is the reduction down to zero of the incidence of a specified disease in a defined geographic area: intervention measures have to be continued as there is a

\section{KARGER \\ Fax +41613061234 \\ E-Mail karger@karger.ch}

www.karger.com (c) 2008 Nestec Ltd., Vevey/S. Karger AG, Basel

0517-8606/08/0662-0055\$24.50/0

Accessible online at:

www.karger.com/ane
Leif Gothefors, Prof. em.

Department of Clinical Sciences/Paediatrics

Umeå University

SE-901 85 Umeå (Sweden)

Tel. +4690785 2102, Fax +46901237 28, E-Mail leif.gothefors@pediatri.umu.se 
Table 1. Deaths from diseases commonly vaccinated against, Australia 1926-2000

\begin{tabular}{lrrrrrl}
\hline Period & Diphtheria & Pertussis & Tetanus & Poliomyelitis & Measles & $\begin{array}{l}\text { Population estimate } \\
\text { millions }\end{array}$ \\
\hline $1926-1935$ & 4,073 & 2,808 & 879 & 430 & 1,102 & 6.6 \\
$1936-1945$ & 2,791 & 1,693 & 655 & 618 & 822 & 7.2 \\
$1946-1955$ & 624 & 429 & 625 & 1,013 & 495 & 8.6 \\
$1956-1965$ & 44 & 58 & 280 & 123 & 210 & 11 \\
$1966-1975$ & 11 & 22 & 82 & 2 & 146 & 13.7 \\
$1976-1985$ & 2 & 14 & 31 & 2 & 62 & 14.9 \\
$1986-1995$ & 2 & 9 & 21 & 0 & 32 & 17.3 \\
$1996-2000$ & 0 & 9 & 5 & 0 & 0 & 18.7 \\
\hline
\end{tabular}

Italics indicate the decade vaccination started for the disease.

Reproduced with permission of the NSW Public Health Bulletin [2].

possibility of reintroduction of the disease from other areas.

A disease can be brought under control, i.e. the reduction of incidence, morbidity or mortality to a locally accepted level; however, continued intervention measures are required to maintain this reduction.

\section{Smallpox}

Through the 19th century, most industrialized countries introduced vaccination, and by 1900 , a number of North European countries had become freed from smallpox. However, vaccination continued until the 1970s as a protective means in case smallpox was reintroduced.

In 1959, the WHO decided to undertake a global eradication programme. Mass vaccination campaigns were introduced but progress was disappointing, especially in Africa and on the Indian subcontinent. Limitations in funding and manpower (WHO staff was at that time preoccupied with malaria eradication) may have contributed to the relative failure. In 1966, the strategy was changed to 'surveillance and containment', also called 'ring vaccination', which includes erecting a barrier of immune persons around the case and his or her contacts [3]. This proved to be a more successful approach, and the last naturally occurring case of smallpox was diagnosed in Somalia in 1977, and subsequently, the WHO declared smallpox as officially eradicated from the world population in 1979. In this situation, would it not be prudent to destroy the known remaining laboratory stocks of the variola virus, e.g., those at the Centre for Disease Control and at the Vector Institute in Novosibirsk? Several scien- tific organizations have recommended destruction of the virus [4], but the issue during the last decade became political. The 2007 World Health Assembly did recommend that the virus stocks (eventually) should be destroyed but not until '... research outcome crucial to an improved public-health response to an outbreak so permit' [5].

In the years after $9 / 11$, the threat of a bioterrorist attack using smallpox as a weapon made the US authorities in 2002 temporarily reinstate smallpox vaccination for military personnel and civilians at high risk. Studies of adverse events probably related to the vaccine showed 21 cases of carditis with 3 deaths in one study (37,901 civilians) and 39 cases of serious neurologic adverse events in another $(665,000$ military and civilian staff) $[6,7]$. However, the prevalence of these diseases in the study population was similar to the expected prevalence among the general population. However, the WHO policy had opposed such pre-event smallpox vaccination, and the Institute of Medicine criticized the Centre for Disease Control for this programme, '... the result of an extraordinary policy decision to vaccinate people against a disease that does not exist with a vaccine that poses some well-known risks' [8].

\section{Tuberculosis}

The annual report 'Global tuberculosis control 2007' [9] concludes that 'the global TB incidence rate peaked sometime between 2000 and 2005 ... the global TB epidemic is now on the threshold of decline'. Today, the main mechanism for achieving this target builds on the success with DOTS (Direct Observed Treatment, Short-course) 
Fig. 1. Diphtheria global annual reported incidence and DTP3 coverage, 1980-2006. Reproduced with permission of WHO.

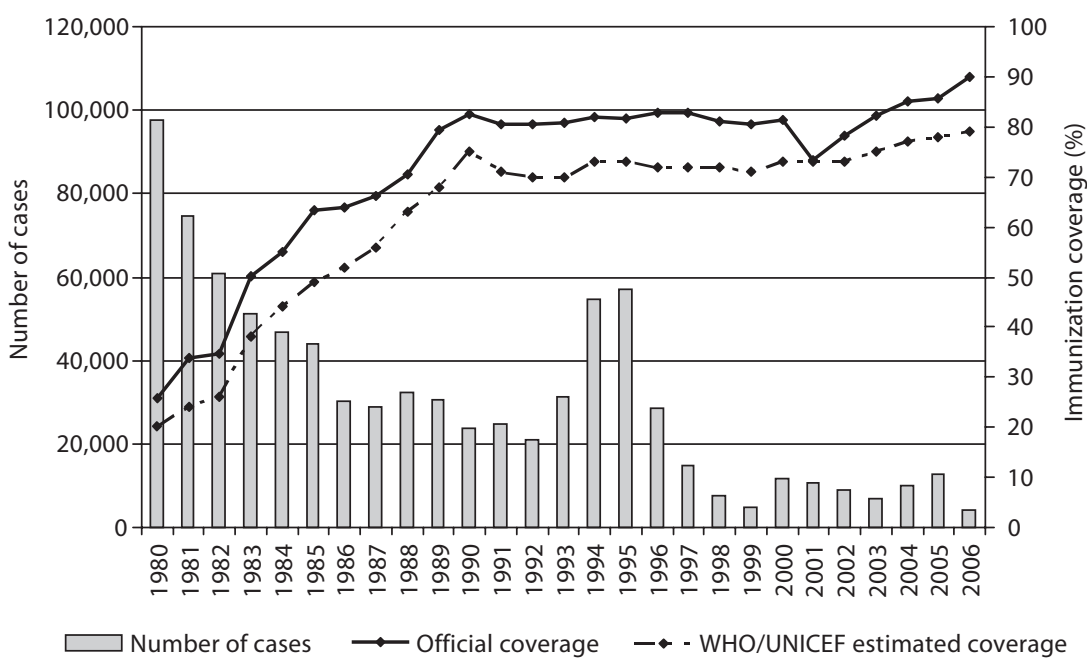

low, BCG is given only to risk groups for the disease or is totally replaced by case detection and early treatment. Neither vaccination of adults nor repeated vaccination is recommended by the WHO [10]. Thus, the BCG vaccine is still 1 of the 3 factors on which TB control is based: early diagnosis and adequate treatment, contact tracing and BCG vaccination. There is an urgent need for a more effective vaccine that could also prevent reactivation of the bacteria.

\section{Diphtheria}

Diphtheria has been one of the most feared childhood diseases, characterized by devastating outbreaks. Fortunately, most infections with Corynebacterium diphtheriae present with a mild clinical course - or are totally asymptomatic - but case fatality rates reached $50 \%$ during a major epidemic in Europe and the US in the 1880s. The fatality rate declined to about $15 \%$ during the First World War (mainly due to the use of antitoxin) and can still reach - in spite of modern treatment - $10 \%$ in endemic areas $[13,14]$. It is estimated that before the vaccine became easily available, about 1 million cases of diphtheria resulting in 50,000-60,000 deaths occurred each year in developing countries [13]. The vaccine became part of the EPI in 1974 and, most probably as a consequence of this, the global number of reported diphtheria cases was reduced by $>90 \%$ (fig. 1) during the period 1980-2000. 
Fig. 2. Neonatal tetanus global annual reported incidence and TT2+ (at least 2 doses of tetanus toxoid) coverage, 19802006. Reproduced with permission of WHO.

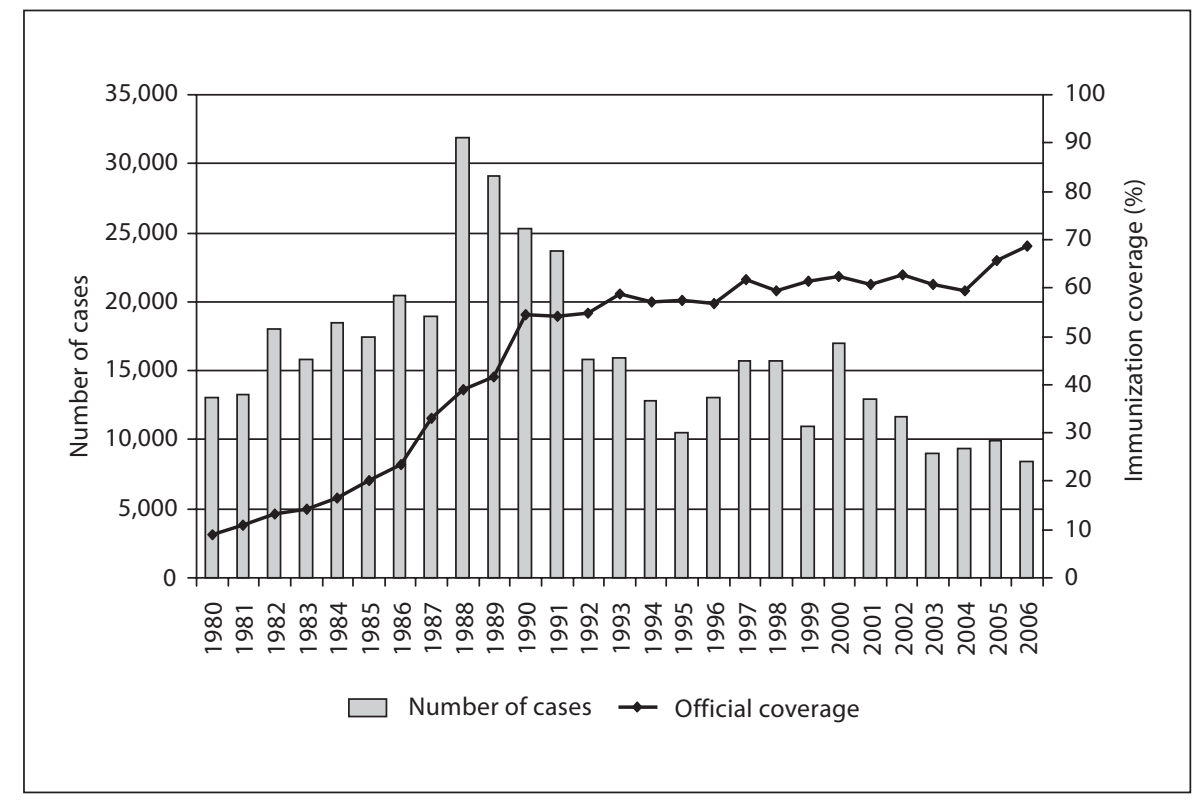

There are countries, particularly in sub-Saharan Africa but also in parts of South America and East Asia, where immunization coverage with 3 doses of diphtheria vaccine (given as diphtheria/tetanus/pertussis whole cell, DTPw) is $<50 \%$ of the birth cohort [13]. In such countries - with diphtheria still endemic - morbidity and mortality due to diphtheria are still high, and preschool and school age children are most commonly affected.

In most industrialized countries - with high vaccine coverage - endemic diphtheria has disappeared or is extremely rare, but outbreaks still occur. Serological studies in the 1980s indicated that even in Europe and the US with high vaccine coverage, a large proportion, often up to $50 \%$, of adults were susceptible to diphtheria [15]. Two important reasons are that (1) vaccine-induced immunity wanes over time unless period booster immunizations are given, and (2) the 'normal' circulation of toxigenic $C$. diphtheriae is reduced by the widespread use of diphtheria vaccine, and thereby, the natural boosting effect on the immune system is reduced [15].

If, in a situation with low vaccine coverage among children and a large gap in the immunity among adults, a new biotype of $C$. diphtheriae would be introduced, an epidemic is possible. Crowding and poor personal hygiene are also important factors. This is probably what happened in the Russian Federation where in the 1990s diphtheria reemerged resulting in an epidemic. The epidemic spread to all Newly Independent States and the Baltic states causing $>157,000$ cases and 5,000 deaths.
Adults accounted for nearly half of the deaths [14, 15]. At least up to 1986, most of these countries had a reasonably high childhood immunization coverage including a booster dose around 15 years of age. However, the immunogenicity of the vaccine used has been questioned. The spread of the epidemic in the Newly Independent States was facilitated by large-scale population movements. This may happen again due to ever increasing international travelling leading to the introduction of toxigenic strains to countries undergoing sociopolitical instability. This instability results in factors believed to be important in producing epidemics such as large numbers of refugees forced to live in extremely difficult hygienic and economic conditions and, importantly, no infrastructure to facilitate the vaccine being distributed to the refugees.

\section{Tetanus}

Tetanus is unique among the diseases prevented by the EPI vaccines: it is not communicable. Unlike smallpox and polio it cannot be eradicated. Its high fatality rate in both industrialized and developing countries, usually associated with injuries in otherwise healthy persons, particularly during military conflicts, made a vaccine desirable. The toxoid vaccine was developed in the 1920s. Effective immunization during World War II reduced the incidence of tetanus cases to about one thirtieth of that reported during World War I [16]. In most developed 
countries, tetanus is now considered rare due to improved wound care and high rates of immunization. The current case distribution reflects incomplete vaccination coverage: thus, most cases occur in the elderly.

However, in the developing world, the health burden from tetanus is largely among the neonates. Aside from that age group, most cases occur among non-immunized boys and young adults where rates of tetanus decline when immunization programmes are in place. In 1980, estimates based on mortality surveys suggested that there were approximately 1 million annual deaths [17] due to neonatal tetanus and 122,000-300,000 non-neonatal tetanus deaths. In 1989, the World Health Assembly called for elimination of (maternal and) neonatal tetanus by 1995. The target date has since been repeatedly postponed. However, progress has been made (fig. 2): the number of deaths in the year 2000 was estimated to 200,000 , and in September 2007, only 47 countries remained that had yet to eliminate maternal and neonatal tetanus. To achieve this goal, a number of strategies are being used [18]: (1) immunizing pregnant women during their ante-natal contacts, (2) immunizing all women in child-bearing age through Supplementary Immunization Activities in areas where women have no or limited access to routine vaccination, and lastly, (3) promotion of skilled care for birth.

\section{Pertussis}

Pertussis was once among the most common childhood diseases. Of all the paediatric infectious diseases for which an effective vaccine given to most infants exists, pertussis is still the most poorly controlled [19]. However, by extensive immunization programmes in the industrialized world beginning in the 1950s, there was a dramatic reduction $(>90 \%)$ in pertussis incidence, and major epidemics were largely eliminated.

The vaccine (as DTPw) has been part of the EPI since the programme started in 1974. Since the end of the 1980 s, about $80 \%$ of all infants worldwide are immunized. In spite of that, the WHO estimated that 17 million cases occurred worldwide in 2003 and that about 279,000 patients died from the disease [20]. However, incidence figures must be interpreted with caution: case definition and surveillance system performance vary greatly between countries.

The WHO recommends primary immunization with DTP to consist of 3 injections given at the age of 6,10 and 14 weeks. Most countries adhere to this schedule, albeit with minor alterations. In many countries, a booster dose is given between 1 and 6 years later. In the Nordic countries and Italy, 3 doses are given at 3, 5 and 11-12 months of age.

Vaccination has had the expected impact among young children where infection now is rare. However, protection following pertussis vaccination wanes after 6-12 years (and so does immunity after natural infection) [20]. Probably, as a result of this, there has been a shift towards a higher incidence of pertussis (symptomatic as well as asymptomatic) among schoolchildren and young adults: $12-21 \%$ of adults with a persistent cough for more than 2 weeks may suffer from pertussis [20,21]. Transmission of the infection from these bacterial reservoirs in older age groups to the very young non-immune infants [22] has resulted in an increasing incidence also of infantile pertussis in many industrialized countries. This is in definite contrast to the aim of the programme, which is to reduce the incidence and severity of the disease among young - and especially among very young - children. To protect these small infants, many being too young to be immunized, the endemicity of pertussis infection could be addressed by boosting immunity in the older age groups [22]. This is possible with the acellular pertussis (aP) vaccine, and the introduction of adolescent immunization is now being discussed in many developed countries [19].

The increasing incidence of pertussis in teenagers and young adults has so far been rarely reported in developing countries or in industrialized countries with low vaccine coverage. In fact, there has been very little documentation about the epidemiology of pertussis in Africa, Asia or Latin America. In Senegal, the same trend was seen over a period of 12 years after introduction of vaccination [23], and serological studies from e.g. Taipei, Israel and Turkey indicate that older children, adolescents and even adults are at risk of pertussis despite a 4-dose immunization programme [23].

The whole-cell pertussis vaccine $(\mathrm{wP})$, produced as DTwP by many manufacturers also in developing countries, has shown an efficacy of $>80 \%$. Due to an increased incidence of minor adverse effects following vaccination and rare neurologic adverse reactions, there was a decreased acceptability of the vaccine in a number of industrialized countries during the 1970s. This led to the development of the acellular pertussis vaccine (known as $\mathrm{DTaP}$ ) which is expected to be efficacious in all regions of the world but is unlikely to be currently affordable in most developing countries [20]. The first priority in order to control pertussis is to reach $90 \%$ global coverage with 
the primary 3 doses of DTwP vaccine. To better understand if the resurgence of pertussis, especially among adolescents and young adults, occurs also in developing nations, an improved surveillance is required.

\section{Polio}

Infantile paralysis, later identified as poliomyelitis, was well known even before the 20th century. At that time, the disease was endemic, with most infections occurring in children 6 months to 4 years of age where nonparalytic meningitis is the most likely consequence of central nervous system involvement. Poor sanitation resulted in a constant exposure to the virus which enhanced a natural and early immunity among the population. In developed countries during the late 19th and early 20th centuries, improvements were made in community sanitation, and paradoxically, this drastically increased the proportion of children and adults at risk of paralytic polio infection by delaying polio virus exposure from infancy to later life [24].

Small localized paralytic polio epidemics were observed in Scandinavia in the late 19th century. The first documented outbreak in Sweden occurred in 1881 in Vaesterbotten county [25], and at the turn of the 20th century, the disease became an annual feature in the Swedish epidemiological pattern. The US saw its first (described) epidemic in 1894 [26].

With further improvement in sanitation, outbreaks increased in size and reached pandemic proportions in Europe, North America, Australia and New Zealand during the first half of the 20th century. By 1950, the peak age incidence of paralytic poliomyelitis in the US had shifted from infants to children aged 5-9 years when the risk of paralysis is greater [27]. The 1952 polio epidemic became the worst outbreak in US history with more than 3,000 dead and 20,000 left with disabling paralysis [28].

In 1907, the Swedish paediatrician Ivar Wickman [29] had categorized the different clinical types of polio and suspected that this was an infectious disease. His theory was strengthened by Landsteiner (Nobel Prize 1930 - but for blood groups) infecting monkeys with spinal cord material from children who had died from the disease. The 3 types were finally identified in 1931 [26].

In 1948 , the same year as the WHO was established, Ender, Weller and Robbins (Nobel Prize 1954) were able to grow the virus in non-nervous tissues. In part based on their findings, Drs. Jonas Salk and Albert Sabin developed their vaccines against polio: an inactivated (killed) injectable (IPV) and a 'live' oral vaccine (OPV). Salk tested his vaccine in one of the world's first double-blind placebo-controlled tests [30]. After a number of years, his vaccine was - in spite of the Cutter incidence [31] where the vaccine was not fully inactivated and caused close to 200 cases of polio - proven to be effective in sharply reducing the number of polio cases, first in the US (1955) and later in many other countries. Soon after Salk's presentation of his vaccine, Swedish scientists [32] presented their own, killed and safer vaccine, but unfortunately, the Cutter incidence delayed its use by several years.

The Sabin vaccine was internationally tested through the WHO in 1959 when large groups in e.g. Russia, Holland, Mexico, Chile, Sweden and Japan received the vaccine [33]. It had the advantage of easier administration, lower price and prevented not only the complications of polio infection but also the initial intestinal infection. It soon became the vaccine of choice for most national immunization programmes in the world. However, a few European countries, e.g., Holland and some Scandinavian countries, continued to use IPV. Sweden introduced IPV in 1957, and already in 1963, polio was almost eradicated in the country. Not only the disease disappeared, but on the whole, the silent circulation of the virus in the community ceased. And this to the great surprise of the advocates of OPV who held the view that OPV gave a much better local immune protection in the intestine, thus preventing the spread of wild poliovirus.

Large polio epidemics had caused panic every summer during the 1940s and 1950s in the US and Western Europe. Sweden was one of the countries first struck by the infection and also had the highest incidence and mortality rate. With the introduction of the vaccine, polio was finally brought under control. It took somewhat longer for polio to be recognized as a major problem in developing countries.

During the 1970s, a large number of lameness surveys (looking for acute flaccid paralysis) demonstrated that polio was abundant also in many developing countries. This led to the introduction of routine immunization with OPV in almost all national EPI programmes. In 1985, the Pan American Health Organization (PAHO) started its initiative to eradicate polio from the American continent. It proved to be a very efficient programme: the last indigenous case of polio occurred in Peru in 1991. Before a region can be declared free of polio, there has to be a 3-year period free of the disease. Thus, the Americas were certified polio free in 1994 [34]. It is interesting that since 1999, North America has returned to the use of IPV due to the risk that the live vaccine virus can revert and 
Fig. 3. Poliomyelitis global annual reported incidence and Pol3 coverage, 19802006. Reproduced with permission of WHO.

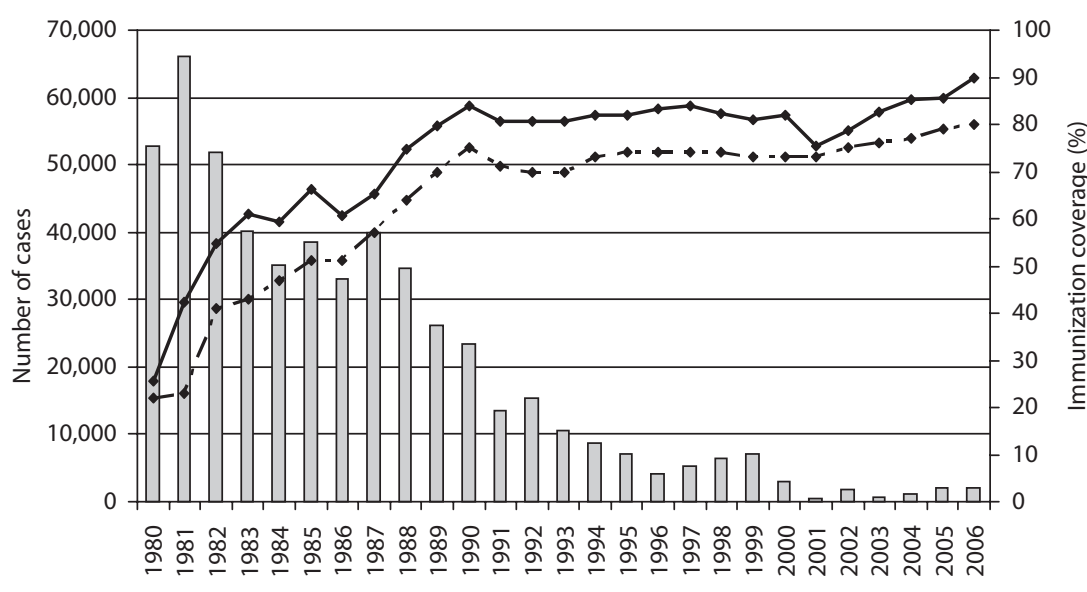

$\square$ Number of cases $\multimap$ Official coverage $\quad \bullet$ - WHO/UNICEF estimated coverage regain its property to cause paralysis: vaccine-associated paralytic poliomyelitis, an inevitable consequence of the use of OPV.

In 1988, poliovirus was endemic in more than 125 countries paralyzing more than 1,000 children every day [26]. That same year, the already positive results from PAHO convinced the World Health Assembly to pass a resolution to eradicate polio by the year 2000 . This statement was in large part based upon the knowledge that funds were made available from the Rotary International and their campaign launched in 1987 to raise USD 120 million to fight polio. By the time the world is certified polio free, their donations will be 5-fold larger [26].

Within the EPI programme, OPV is usually administered with 4 doses before the child's first birthday. By 1990 , almost $80 \%$ of children born each year were immunized, and there has been a substantial drop in the incidence of the disease (fig. 3).

In order to further improve the situation, National Immunization Days (NIDs) were initiated: these mass immunization campaigns are aimed at complementing, not replacing, routine immunization. The goal is to interrupt the circulation of poliovirus by having every child under 5 years of age immunized with 2 doses of OPV, 1 month apart, regardless of the previous immunization status. This way, each child in the most susceptible age group is protected against polio at the same time, i.e. the virus has no host in which to survive. Volunteers with minimal training can act as vaccinators, an example being an NID in India where 2 million volunteers immunized 134 million children on a single day [26].

China had a large outbreak in 1989-1990 with 5,000 cases each year that eventually led to NIDs in 1994, where 80 million children were immunized within a short period [26]. Two years later, the last case of polio in China was identified. India followed the example with their first NID in 1995.

The activities in the WHO Western Pacific region led to this region being certified free from polio in the year 2000 - the last case was seen in Cambodia in 1997. The WHO Western Pacific region includes China as well as Indonesia with more than a quarter of the total global population [35].

Often 3-5 years of repeated NIDs (in endemic areas 7-8 rounds per year) are required to eradicate polio, but some countries need more time, especially those where routine immunization coverage is low. India and China are good examples of individual country activities. However, the polio virus does not respect borders, and thus, neighbouring countries are trying to 'synchronize' their NIDs. This approach was used in 1995 among 19 countries of Eastern Europe and Central Asia (Operation MECACAR: Mediterranean, Caucasus, Central Asian Republics and Russia) with 56 million children immunized [26]. Similar multinational campaigns have been carried out in Western and Central Africa (in the year 2000, 76 million, and in 2004, 63 million children) and along the border of Afghanistan and Pakistan and other 
Table 2. Confirmed cases of polio caused by wild virus in endemic and non-endemic countries in the years 2000-2007 [26]

\begin{tabular}{|c|c|c|c|c|c|c|c|c|}
\hline & 2000 & 2001 & 2002 & 2003 & 2004 & 2005 & 2006 & 2007 \\
\hline \multicolumn{9}{|c|}{ Cases in currently endemic countries (Nov 2007) } \\
\hline India & 265 & 268 & 1,600 & 225 & 134 & 66 & 676 & 873 \\
\hline Nigeria & 28 & 56 & 202 & 355 & 782 & 830 & 1,122 & 285 \\
\hline Pakistan & 199 & 119 & 90 & 103 & 53 & 28 & 40 & 32 \\
\hline Afghanistan & 27 & 11 & 10 & 8 & 4 & 9 & 31 & 17 \\
\hline \multicolumn{9}{|c|}{ Total cases in endemic countries } \\
\hline & 702 & 475 & 1,915 & 732 & 999 & 943 & 1,869 & 1,207 \\
\hline \multicolumn{9}{|c|}{ Total cases in non-endemic countries } \\
\hline & 17 & 8 & 3 & 52 & 256 & 1,036 & 125 & 106 \\
\hline \multicolumn{9}{|l|}{ Countries, $\mathrm{n}$} \\
\hline & 23 & 15 & 9 & 15 & 18 & 16 & 17 & 12 \\
\hline & 20 & 10 & 7 & 6 & 6 & 6 & 4 & 4 \\
\hline
\end{tabular}

Data in WHO HQ as of 21 May 2008.

zones of war and conflict where ceasefire was negotiated for a few days of immunization activities [26].

The year 2000 saw a record 550 million children, almost $1 / 10$ of the world population, receive OPV [26], and in the same year, 719 cases of wild poliovirus meant a $99 \%$ reduction since the programme started in 1988 (table 2).

In Europe, barely 400 cases in the year 1990 were reduced to only 200 in 1992 . That year, a polio outbreak in the Netherlands (with the virus spread from India via the Middle East) occurred among a group who for religious reasons refused to be immunized, and thus, the virus from that epidemic spread to Canada [26]. Small epidemics in Azerbaijan and Ukraine occurred in 1993, and Albania, which had been free of wild poliovirus for 18 years, had an outbreak in 1996 which spread to neighbouring countries [26]. Finally, Turkey had the last indigenous polio case in Europe in 1998, and in 2002, the WHO European region (51 countries) was certified as freed from polio [36].

Obviously, the polio campaign for eradication of the virus has been very successful, but it did not come to an end in 2000 as planned. The time for conclusion has been repeatedly postponed.

There are a number of explanations for this delay: infection with poliovirus causes symptoms from the central nervous system only in a small fraction of those infected, translating to about 1 case per 100-200 infected. It is consequently difficult to follow and control the massive spread of the virus from many, usually silent intestinal infections.
There are also some problems with the existing vaccines: both vaccines have to be given in repeated doses and the 3 types of poliovirus compete with each other. To get an effective multiplication of all 3 types, the vaccines have to be given at least 3 times. Other enteric viruses can also compete with the vaccine virus: the live vaccine can show problems in areas with poor hygiene. Another problem with the live vaccine is the possibility of reversion. Vaccine-derived polioviruses (VDPVs) can (1) produce polio outbreaks in areas with low rates of OPV coverage ('circulating' cVDPVs) and (2) replicate for years in immunodeficient persons (iVDPVs). When the vaccine virus is spread among many non-immune persons and along the way reverts to wild poliovirus, it can cause paralysis in unimmunized persons. cVDPVs can be prevented from emerging by good vaccine coverage. iVDPV, on the other hand, can arise any time a person with a primary immunodeficiency is exposed to OPV. The only way to prevent new iVDPV infections is to stop OPV use.

The year 2003 looked promising (table 2), and there were only 784 new cases registered, almost half of them in Nigeria. In spite of continued strong efforts, it was by now obvious that the last remnants of polio would be difficult to eliminate. However, the number of countries where polio is endemic has now been reduced to 4: Afghanistan, Pakistan, India and Nigeria, and these 4 countries act as source of infection for new introduction in countries which earlier were declared free from polio (fig. 4). 


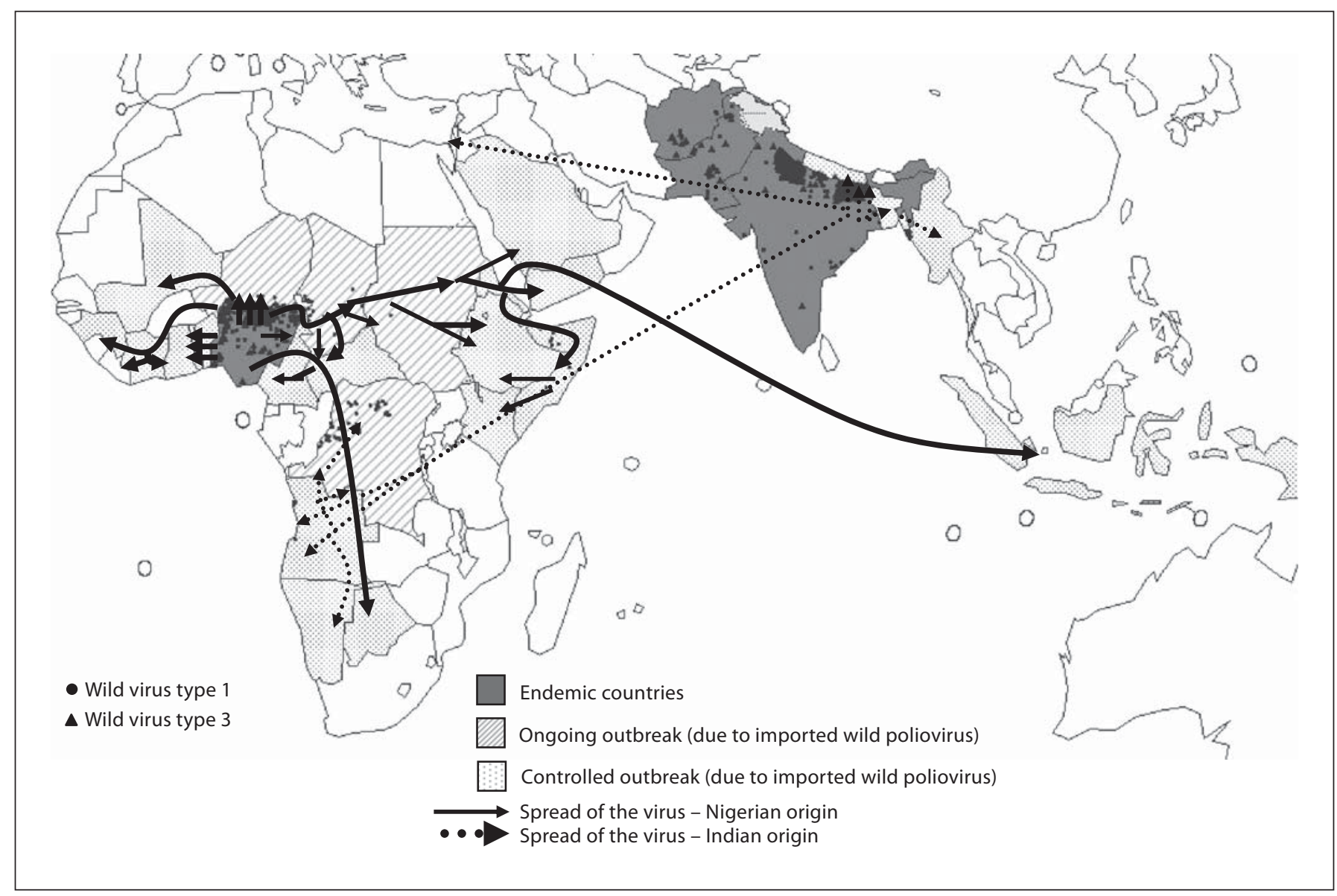

Fig. 4. Wild poliovirus spread, 2003-2007 (excluding viruses detected from environmental surveillance and vaccine-derived polio viruses). Endemic countries: as of 1 January 2006, Egypt and Niger were reclassified from endemic to non-endemic. Reproduced with permission of WHO.

In August 2003, a number of northern states in Nigeria suspended polio immunization campaigns following concerns by some public figures regarding the safety of the polio vaccine. Subsequently, a new outbreak occurred, originating in the state of Kano, thus reinfecting previously polio-free areas within Nigeria as well as 8 previously polio-free countries across West and Central Africa (table 2; fig. 4) [37]. When immunization activities were reintroduced in Northern Nigeria in July 2004, the largest-ever multi-country campaign was performed where more than 1 million vaccinators in 23 countries across West and Central Africa took part and more than 80 million children were immunized. The number of cases continued to increase in Nigeria, but in 2007, there seems to be an improvement (table 2). Nigeria remains the single largest risk to the aim of polio eradication [38]. There is widespread transmission due to gaps in population im- munity, and in several of the northern states, more than $20 \%$ of children $<5$ years old have not received a single dose of OPV [39].

In 2002, India had a large epidemic with 1,600 cases. However, in 2005, with dramatically increased quality as well as quantity of immunization campaigns, India could report the lowest number of cases ever (66 cases). Western Uttar Pradesh had an outbreak in 2006 aided by high population density and poor sanitation. It is also possible that the high prevalence of enteric infections can interfere with immune response and explain the low efficacy of OPV compared with other parts of India [38]. As a result, most children in Uttar Pradesh received more than 12 doses of vaccine before their 2 nd birthday to attain immunity [39].

In Pakistan, transmission is restricted to areas where security is compromised, and the situation is similar in 
southern Afghanistan with a significant outbreak in 2006. In the border areas between the 2 countries, political instability and armed conflicts make immunization activities very difficult [38].

The outbreaks in most of the 24 countries were successfully stopped in 2005 (at a cost of more than USD 450 million) (table 2). However, the largest single-country epidemics in recent years in Yemen and Indonesia were not fully stopped until 2006.

The polio virus type 2 has not been found since 1999 , and as a consequence, monovalent vaccines with either type 1 or type 3 are now used. Studies have shown that monovalent mOPV1 is 3 times more effective than trivalent OPV in protecting children against polio [37]. It has been successfully used in endemic areas such as Uttar Pradesh. mOPV 3 has similarly been used in areas where serotype 3 dominates.

Due to these widespread epidemics in previously polio-free countries in 2003-2006, scepticism was expressed about the achievability of eradication. Maybe continued 'effective control' (defined as maintaining $<500$ polio cases/year indefinitely) would be cheaper and more attainable [26]. However, today, there are new tools (improved rapid diagnostics and more potent monovalent vaccines) which have contributed to the substantial reduction in polio cases in 2007 (table 2). Total eradication is still (2008) the goal. Success depends on the remaining 4 countries where tailored approaches for each of them are needed due to different operational challenges.

If the world eventually is certified as polio free, what then? The risk of resurgence is still a reality. One problem is the large proportion of subclinical infections which facilitates a silent spread of the virus. Another problem is the above mentioned possibility that the live vaccine virus (OPV) can revert and cause paralysis: 1 case in 3 million vaccinated and at least 9 small outbreaks reported (caused by cVDPVs) with totally 200 paralyzed infants [26]. It will be critical to maintain population immunity over a long term to prevent circulation of re-emerging viruses. In September 2006, the Advisory Committee on Poliomyelitis Eradication gave the following recommendations for the eventual cessation of routine immunization with OPV [40]: (1) confirmation of the interruption and containment of wild poliovirus globally; (2) use of highly sensitive surveillance; (3) establishment of stockpile of monovalent OPV for responding to reintroduced circulating poliovirus; (4) maintenance of a $>90 \%$ nationwide coverage of IPV in all countries with poliovirus facilities; these facilities (which could conduct essential reference and research functions) should be less than 20 worldwide; (5) synchronous global cessation of the use of OPV for routine immunization, and (6) containment of Sabin poliovirus strains.

However, such a prolonged campaign will be costly: USD 400 million per year until virus eradication and several years thereafter. Nonetheless, the task has to be finished; the world is only small steps away from ridding itself of polio forever.

\section{Measles}

Infection with measles virus is second to malaria in terms of the number of people who die each year as a result of complications from an infectious disease [41].

The first text on measles from the 10th century was written by the Persian physician Rhazes. For many centuries, it was then supposed that measles and smallpox were closely related: 'measles more to be dreaded than smallpox' [42]. In the 17th century, the distinction between the two was made, and in 1758, the infectious nature of measles was recognized by Francis Home. The epidemiology was much better understood after the Faroe Islands epidemic in 1846 [42]. In 1954, Enders and Peebles managed to isolate the virus in tissue cultures, and the vaccine was licensed in 1963.

Measles occurs in epidemic cycles, and in developed countries usually starts in large cities, the reservoirs of measles where it remained endemic, and then spreads to the countryside where transmission could die out after an epidemic and the virus has to be reintroduced for the next epidemic season to begin.

Before the introduction of the vaccine, the risk of infection in developed countries was the highest in school age children. On the other hand, in many developing countries, infection occurred at a much lower age. In Africa, $100 \%$ of 4 -year-old children were expected to have had measles. There, the children are exposed to the community at large at an early age (at least compared with children in developed countries) with a high risk to be infected. Additionally, their often suboptimal immunity due to e.g. poor nutrition (vitamin A) and the rapid loss of placentally transferred antibodies explains why measles infection strikes so early and often is so severe in developing countries. Crowding, leading to a high dose of the virus, may also be important [43].

When the vaccine was introduced, the interval between epidemics increased while the size of them was reduced. Relatively more cases were also seen among older children who either never had been vaccinated or had 
Fig. 5. Measles global annual reported incidence and measles vaccine coverage, 1980-2006. Reproduced with permission of WHO.

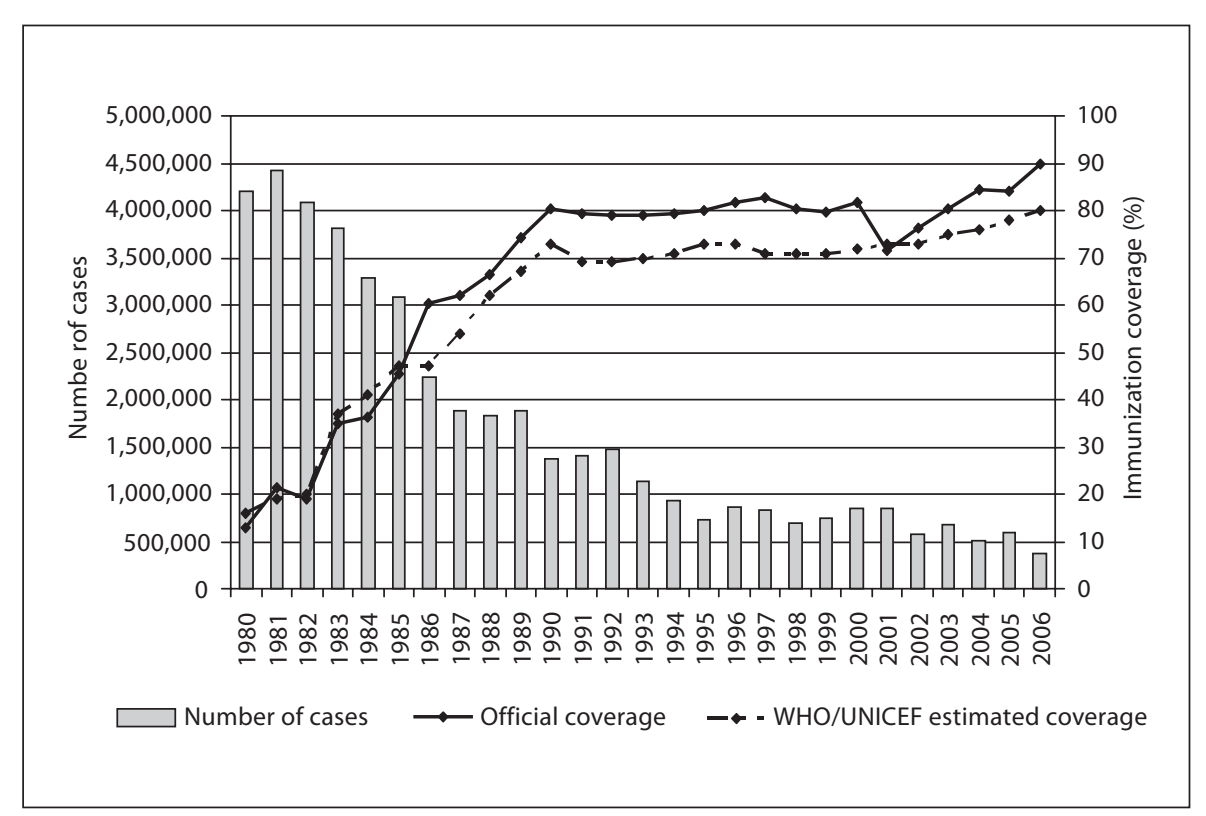

failed to respond to the vaccination. When coverage increases and viral transmission decreases, even unvaccinated individuals have a reduced risk of infection. It has been estimated that the threshold for herd immunity is obtained with $93-95 \%$ vaccine coverage [44]. When this immunity is maintained, transmission of the virus will die out and measles is eliminated. Still, a country can have cases imported from other countries where measles is still endemic. However, in a well-immunized society, those cases will not cause more than a few secondary cases. When measles is eliminated (at the same time) in every country, there is global eradication.

In the western world, a single dose of vaccine given at 12-18 months of age induces immunity in about $95 \%$ of vaccinees. When the $5 \%$ who fail to respond to the first dose receive a second dose, $>95 \%$ do respond, and thus, with $95 \%$ coverage with 2 doses, the target for herd immunity can be reached [41].

For developing countries, immunization was recommended already at 9 months of age due to the high morbidity and mortality among infants. At that age, seroconversion occurs in only $85 \%$ [41]. With this rate of conversion and a 90\% coverage (a quite optimistic figure for many countries), only $77 \%$ of the children would be immune. Even a second dose at an age when seroconversion is $95 \%$, given within the routine health services with only $90 \%$ coverage (or less), would not much improve the situation regarding herd immunity. The answer to the problem could be mass campaigns: if during a campaign, $90 \%$ of previously unvaccinated children get their first dose and $90 \%$ of those previously vaccinated will get their 2 nd dose, population immunity will be greater than the necessary $95 \%[41,45]$.

The vaccine was gradually introduced through the EPI from 1974 in almost every country in the world with the recommendation from the WHO/UNICEF that 1 dose of vaccine should be given at 9 months of age to at least $80 \%$ of children in every country. Towards the end of the 1980 s, global coverage was close to $80 \%$ (fig. 5), but then, the interest among the donors decreased, and thus, the coverage levelled out at $70-80 \%$ during the 1990 s. The problem was not only that low coverage kept the number of measles cases high; as mentioned, usually young infants were infected and case mortality was high. How can we protect these young infants?

Could infants be vaccinated at an even younger age? To overcome the neutralizing effect of maternal antibodies, a high-titre measles vaccine was developed and given at 4-6 months of age in a number of African countries. The vaccine was more immunogenic than the standard vaccine, protected against measles in that age group, but, was associated with an unexpected increase in number of deaths (diarrhoea, pneumonia and malaria) among females. Those vaccines are not used today [46].

PAHO developed another approach. Towards the end of the century, many industrialized countries had followed the examples of Sweden and Finland and introduced a second routine dose. In 1987, Cuba performed a 
onetime nationwide mass vaccination campaign with the aim to immunize all children from 9 months to 14 years of age regardless of earlier vaccination history. The idea was that transmission among older children should be reduced and thereby transmission to infants would also be prevented. The strategy, successful in Cuba, was introduced to the rest of the Americas and later to the rest of the world [47]. It has 3 components: (1) maintain $>90 \%$ coverage in routine immunization at 9-12 months of age in every district, (2) perform a onetime mass vaccination of all children 1-14 years, and (3) repeat mass campaigns every 3-5 years for all children 1-5 years old, irrespective of prior disease or vaccination history. Effective surveillance for cases of measles and good clinical management of patients with measles is of course also important. In the Western Hemisphere, this strategy resulted in a $>99 \%$ decline in reported cases during the 1990s. In 2002, Venezuela and Colombia were the only countries with large outbreaks, and in September 2003, PAHO announced that the Western Hemisphere had been free of endemic measles for 10 consecutive months [41].

In 2002, the UN General Assembly Special Session on Children set a goal to reduce worldwide measles mortality by $50 \%$ from the year 1999 to the end of 2005 . Recent modelled estimates indicate that in the named period, there actually was a gratifying $60 \%$ decrease, mainly through activities in the African region [48]. These estimates gain support from reports from countries where recommended vaccination strategies have been implemented. A 92\% reduction in reported measles cases was seen in 19 African countries. In one of the world's poorest countries, Malawi, the number of measles cases plummeted from 7,000 in 1997 to only 2 cases in 1999 [49]. Cambodia and Vietnam reported a decline in cases from 12,237 and 16,512 to 264 and 410, respectively, during a 5 -year period [48].

The WHO and UNICEF with partners have now a vision for the period 2006-2015: 90\% coverage in every district and $90 \%$ reduction in worldwide measles mortality between 2000 and 2010. Large countries like India, Pakistan and Indonesia still have a high burden of measles cases and deaths, and there, the activities described above have to be fully implemented $[48,49]$.

In countries where high coverage of routine vaccination has been achieved, the second opportunity for measles immunization can instead be provided through a routine 2-dose measles vaccination where the second dose is given at 12-18 months of age or at school entry. This has been the schedule introduced by most industrialized countries, especially after having experienced re- surgence of measles. This happened in the US between 1989 and 1991 with more than 50,000 reported cases and 123 deaths, where a low vaccine coverage was the most important reason for the outbreak [41]. In the mid 90s, Canada with a 1-dose programme had an outbreak of measles in older children and young adults [41]. The vaccine coverage was $>90 \%$ (but not $100 \%$ ) so the small proportion of children who remained susceptible, either as a result of primary vaccine failure or of not having received the first dose, had accumulated over time. When the virus was introduced, the group was large enough to allow transmission, and an outbreak occurred. Experiences from other countries (South Korea, Brazil and Sri Lanka) have confirmed that a single-dose strategy cannot prevent episodic outbreaks of measles [41].

Outbreaks in Europe also remain common although deaths are now rare. In 2004, over 29,000 cases were reported in the WHO European region. The controversy over the safety of the measles vaccine in the late 1990s caused a declining uptake in many countries. In London, as many as $44 \%$ of preschool children were at one time susceptible, prompting vaccination campaigns to be implemented. Coverage improved but is still (at the beginning of 2007) alarmingly low, as e.g. in London: $77 \%$ for the first dose and 52\% for the second dose [50, 51]. Large outbreaks are seen in the UK, spreading to other European countries. Other examples: in 2000, Holland and Ireland had outbreaks with 3,300 and 1,200 cases, respectively, and in each country $>100$ hospitalized and 2-3 deaths. In 2006, Nordrhein-Westfalen (Germany) had more than 1,000 cases; $15 \%$ of them needed hospitalization [52].

Can measles be eradicated? Would it not be more costeffective to maintain high immunization coverage and prevent measles deaths? The benefits would be savings on treatment of patients with measles and savings from stopping measles surveillance and vaccination. However, a number of factors speak in favour of eradication: (1) there is no animal reservoir, (2) chronic shedding of the virus (as with polio, herpes or viral hepatitis) has not been described, (3) good diagnostic tests are available, (4) vaccination is safe and effective, and (5) viral transmission has been possible to interrupt in large geographic areas. But, there are also problems: increasing population density in metropolis areas requires high coverage; ongoing war and terrorist acts prevent immunization activities; increasing international travel, which facilitates the spread of viruses. Even if measles virus transmission can be interrupted all over the world, there is still the threat of reintroduction from laboratory accidents or biological 
weapons. Measles immunization is also reported to overall reduce child mortality, not only through avoidance of measles but through protection against conditions other than measles [53]. Most probably, we will have to continue forever with at least a 1-dose schedule.

\section{Remaining EPI Vaccines}

The WHO has recommended the inclusion of the following vaccines in routine infant immunization:

1988 yellow fever vaccine in countries at risk for the disease

1992 hepatitis B vaccine globally

2006 Haemophilus influenzae type b (Hib) vaccine globally 'unless robust evidence exists of low disease burden'

2006 pneumococcal conjugate vaccine 'should be considered for inclusion in countries where pneumococcal invasive disease is considered a public health problem and where the vaccine serotypes match the most important local serotypes'.

\section{Yellow Fever}

Yellow fever, once a much feared epidemic disease, has been under control for almost 40 years through mass vaccination campaigns. However, since the late 1980s, the disease has returned with new force, in sub-Saharan Africa and South and Central America. Usually, the disease causes sporadic cases or small outbreaks, but as the appropriate mosquito vector is returning to cities where it once was eradicated, there is also the risk of the devastating form of urban epidemics. Appropriate vectors also exist in Asia. Mass prevention vaccination campaigns are now supplementing the routine immunization of infants [54].

\section{Hepatitis $B$}

Three hundred and fifty million people are chronic carriers of the virus, and 500,000-700,000 people die each year from liver disease (cirrhosis or cancer) [55]. The vaccine, available since 1982, is 'the first vaccine against a major human cancer' and it is very safe and induces protection in about $95 \%$ of healthy people, especially in infants and children. Eighty-five percent of all countries have included the vaccine in their programme, and more than 1 billion doses have been given. The prevalence of hepatitis $\mathrm{B}$ virus (HBV) infection and chronic carrier state has effectively been reduced. An example: the chronic carrier rate in Taiwanese children has been reduced from $9.8 \%$ before the HBV immunization programme (1984) to $0.7 \% 15$ years after the programme (1999). The incidence of childhood hepatocellular carcinoma has also declined in Taiwan, but only from 0.54 to 0.20 (per 100,000 children) [56]. The vaccine is very effective in preventing horizontal transmission, while the maternal transmission cannot be interrupted completely by the current HBV immunization programme. The lack of hepatitis B immunoglobulin injections to infants of highly infectious mothers is an important cause of this (relative) failure. The cost of immunoglobulin may be prohibitive in many countries; however, the cost of hepatitis $B$ vaccine (which when introduced cost USD 100 per dose in industrialized countries) is now similar to that of the traditional vaccines in the EPI programme, i.e. USD 0.25 per dose.

\section{Haemophilus influenzae Type $b$}

During the 1990s, the Hib vaccine was introduced into routine infant immunization services by most industrialized countries, and diseases caused by Hib (pneumonia and meningitis) were practically eliminated. In general, a 3-dose primary series is given at the same time as DTP. In the UK, an accelerated 2-3-4 months schedule was used. In spite of good vaccine coverage, in 1999-2002, there was an increase in invasive disease indicating a need for a booster in the second year of life [57].

Developing countries have been hesitant to introduce this vaccine because of its relatively high price and the problems to establish the burden of Hib disease in areas with poor surveillance. However, with financial support from the Global Alliance for Vaccines and Immunization (GAVI), more than 20 developing countries have now managed to introduce the vaccine. Data from Kenya, where the vaccine was introduced in 2001, indicate that the Hib vaccine is a highly cost-effective intervention and could be cost-saving if the vaccine price was below half of its present level [58].

\section{Pneumococcal infections}

Pneumococcal infections (pneumonia and meningitis) are estimated to kill 1.3 million children under the age of 5 every year. Nearly all these deaths occur in the world's poorest countries. There is today (2008) only 1 vaccine licensed for use in infants and young children, a conjugate vaccine including the 7 most common serotypes in the US (10- and 13-valent vaccines are under development). It was introduced in the US in 2000 and has caused a rapid drop in the rate of invasive infections caused by the vaccine types in vaccinated children but 
also in unvaccinated children and adults demonstrating herd immunity [59].

GAVI as well as other public-private partnerships are very active in supplying pneumococcal vaccines to the developing world. Their sincere efforts are commendable, but Ministries of Health are advised - before deciding on introduction of the vaccine - to consider a number of problems related to its use. An area of concern is the observation of an increase in non-vaccine serotype disease, and there has been a reported increase in antibiotic resistance in these 'replacement strains'. The serotype distribution also varies from one country to another: serotypes 1 and 5 together are thought to be responsible for $29 \%$ of invasive pneumococcal disease in India, but the heptavalent vaccine does not cover these serotypes [60].

\section{Epilogue}

Vaccinology can claim to be one area of modern medicine that has led to the spectacular increase in life expectancy in the last 2 centuries. However, as this short survey has shown, there are still a number of issues to be solved before the actual infections are eliminated or at least under control.

Through the eminent work of the EPI, in 1990, the global vaccination rate was thought to be $80 \%$ but has since levelled out (or even dropped in some countries) with a small increase during the last few years. This translates to $15-25 \%$ of children being still unimmunized worldwide. The international community must continue to devote the necessary resources needed to reach these unfortunate children. Perhaps it is more important to strengthen the national EPI programmes - or even better, the national health systems - in order to achieve high coverage than to introduce new, relatively expensive vaccines with sometimes questionable relevance.

The current immunization schedule $(6,10$ and 14 weeks) was developed in the early 1980s for DTP-OPV. With a better understanding of immune responses and the importance of herd immunity, there may be more optimal schedules, at least for some antigens (Hib and pneumococcal). If such schedules allow for fewer doses to be given, it would be of great practical as well as economical advantage. The Nordic vaccination schedule (3 and 5 months in a primary series plus a booster at 12 months) could perhaps be applied in developing countries a well.

\section{Acknowledgements}

The following colleagues have given valuable comments on the manuscript: Rashmi Rodriguez, Cecilia Young, Rose-Marie Carlsson, Bo Stensson and Hans K:son Blomquist.

\section{References}

1 Medical milestones: poll results. BMJ, Jan 6, 2007. www.bmj.com/cgi/content/full/334/ suppl_1/DC3.

2 Burgess M: Immunisation: a public health success. NSW Public Health Bull 2003;14:1-5.

3 Henderson DA, Borio LL, Lane JM: Smallpox and vaccinia; in Plotkin SA, Orenstein WA (eds): Vaccines. Philadelphia, Saunders, 1999, pp 123-154.

4 WHO: Report of the Ad Hoc Committee on Orthopoxvirus Infections. Executive Board report EB/95/33, Oct 10, 1994.

5 World Health Assembly, item 12:2, Aug 18, 2007. www.who.int/gb/ebwha/pdf_files/ WHA60/A60_R1-en.pdf.

-6 Casey CG, Iskander JK, Roper MH, et al: Adverse events associated with smallpox vaccination in the United States, January-October 2003. JAMA 2005;294:2734-2743.

-7 Sejvar JJ, Labutta RJ, Chapman LE, et al: Neurologic adverse events associated with smallpox vaccination in the United States, 2002-2004. JAMA 2005;294:2744-2750.
8 Institute of Medicine/Committee on Smallpox Vaccination Program Implementation: The Smallpox Vaccination Programme: public health in an age of terrorism. March 3, 2005. www.nap.edu/catalog.php?record id $=11240$ \#toc.

9 Global tuberculosis control - surveillance, planning, financing. WHO report 2007. WHO/HTM/TB/2007.376. www.who.int/tb/ publications/global_report/2007/introduction/en/index.html.

10 BCG vaccine. WHO position paper. Wkly Epidemiol Rec 2004;79:27-38.

11 Weill-Halle B: Oral vaccination; in Rosenthal SR (ed): BCG Vaccination against Tuberculosis. Boston, Little, Brown, 1957, pp 175-182.

12 Safety of BCG vaccine to HIV infected children. Global Advisory Committee on Vaccine Safety. Wkly Epidemiol Rec 2007;82: $18-24$.

13 Diphtheria vaccine. WHO position paper. Wkly Epidemiol Rec 2006;81:24-32.
14 Wharton M, Vitek CR: Diphtheria toxoid; in Plotkin SA, Orenstein WA (eds): Vaccines. Philadelphia, Saunders, 1999, pp 211-228.

15 Mattos-Guaraldi AL, Moreira LO, Damasco PV, Hirata Junior R: Diphtheria remains a threat to health in the developing world - an overview. Mem Inst Oswaldo Cruz 2003;98: 987-993

16 Long AP: Immunization to tetanus. Workshop on professional medical experiences in Japan and Korea 1950-53. Walter Reed Army Medical Center, April 29, 1954.

17 Wassilak SGF, Roper MH, Murphy TV, Orenstein WA: Tetanus toxoid; in Plotkin SA, Orenstein WA (eds): Vaccines. Philadelphia, Saunders, 1999, pp 745-781.

18 Maternal and neonatal tetanus (MNT) elimination: the initiative and challenges. www. who.int/immunization_monitoring/diseases/MNTE_initiative/en/index.html.

19 Plotkin S: Aims, scope and findings of the global pertussis initiative. Pediatr Infect Dis J 2005;24:S5-S6. 
-20 Pertussis vaccines. WHO position paper. Wkly Epidemiol Rec 2005;80:31-39.

21 Singh M, Lingappan K: Whooping cough. The current scene. Chest 2006;130:15471553.

22 Tan T, Plotkin S: Controlling pertussis: considerations for the future. Pediatr Infect Dis J 2005;24:S98.

23 Tan T, Trindade E, Skowronski D: Epidemiology of pertussis. Pediatr Infect Dis J 2005 24:S10-S18.

24 Robertson S: Module 6: Poliomyelitis. The Immunological Basis for Immunization Series. WHO/EPI/GEN/93.16. Geneva, WHO, 1993.

25 Axelsson P: The history of polio in Sweden - from infantile paralysis to polio vaccine. Sven Med Tidskr 2004;8:57-65.

26 www.polioeradication.org.

27 Melnick JL: Poliomyelitis; in Warren K (ed): Tropical and Geographical Medicine, ed 2. New York, McGraw-Hill, 1990, pp 558-576.

28 Zamula E: A new challenge for former polio patients. FDA Consumer 1991;25:21-25.

29 Cohen MJ, Kaufman SR, Reiner BD: Aping science - a critical analysis of research at the Yerkes Regional Primate Research Center. Persp Med Res 1995;5:21-22. www.curedisease.com/Faqspolio.html.

30 Francis T, Korns R, Voight R, et al: An Evaluation of the 1954 Poliomyelitis Vaccine Trials. Ann Arbor, University of Michigan, 1955.

31 Offit PA: The Cutter incident, 50 years later. N Engl J Med 2005;352:1411-1412.

32 Wesslén T, Lycke E, Gard S, Olin G: Inactivation of poliomyelitis virus by formaldehyde. Arch Gesamte Virusforsch 1957;7:125-135.

33 Pan American Health Organization: Proceedings of the 2nd International Conference on Live Poliovirus Vaccines. Washington, PAHO, 1960.

34 International notes certification of poliomyelitis eradication - the Americas 1994. Morb Mortal Wkly Rep 1994;43:720-722.
5 General news. Major milestones reached in global eradication: Western Pacific region is certified polio-free. Health Educ Res 2001; 16:109.

36 European region of the World Health Organization. Europe achieves historic milestone as region is declared polio-free. Press release, June 21, 2002.

37 Progress towards poliomyelitis eradication in Nigeria, January 2005 to December 2006. Wkly Epidemiol Rec 2007;82:105-116.

38 Conclusions and recommendations of the Advisory Committee on Poliomyelitis Eradication, Geneva, 11-12 October, 2006. Wkly Epidemiol Rec 2006;81:453-464.

9 Pallansch MA, Sandhu HA: The eradication of polio - progress and challenges. N Engl J Med 2006;355:2508-2511.

40 Conclusions and recommendations of the Advisory Committee on Poliomyelitis Eradication, Geneva, 11-12 October, 2006. Wkly Epidemiol Rec 2006;81:465-468.

41 Meissner HC, Strebel PM, Orenstein WA: Measles vaccines and the potential for worldwide eradication of measles. Pediatrics 2004; 114:1065-1069.

42 Drutz JE: Measles: its history and its eventual eradication. Semin Pediatr Infect Dis 2001;12:315-322.

43 Strebel PM, Papania MJ, Halsey NA: Measles vaccine; in Plotkin SA, Orenstein WA (eds): Vaccines. Philadelphia, Saunders, 1999, pp 389-440.

44 Cutts FT, Steinglass R: Should measles be eradicated? BMJ 1998;316:765-767.

$\checkmark 45$ Measles vaccine: WHO position paper. Wkly Epidemiol Rec 2004;79:130-142.

46 Expanded Programme on Immunization. Safety of high-titre measles vaccines. Wkly Epidemiol Rec 1992;67:357-361.

47 Measles: mortality reduction and regional elimination. Strategic plan 2001-2005. $\mathrm{WHO} / \mathrm{V} \& \mathrm{~B} / 01.13$.
48 Wolfson LJ, Strebel PM, Gacic-Dobo M, et al: Has the 2005 measles mortality reduction goal been achieved? A natural history modelling study. Lancet 2007;369:191-200.

49 Davey S: Measles eradication still a long way off. Bull World Health Organ 2001;79:584585 .

50 NHS Immunisation Statistics England 2006-2007. www.ic.nhs.uk/statistics-anddata-collections/health-and-lifestyles/immunisation.

51 Ashmore J, Addiman S, Cordery R, Maguire $\mathrm{H}$ : Measles in North East and North Central London, England: a situation report. Euro Surveill 2007;12E070920.2. www.eurosurveillance.org/ew/2007/070920.asp\#2.

52 van Treeck U: Measles outbreak in Germany: over 1,000 cases now reported in Nordrhein Westfalen. www.eurosurveillance.org/ew/ 2006/060511.asp.

-53 Shann F: A little bit of measles does you good. BMJ 1999;319:4-5.

54 www.who.int/csr/disease/yellowfev/global partnership/en/index.html.

55 www.path.org/vaccineresources/hepb-indepth.php.

56 Chang M, Chen TH, Hsu HM, et al: Prevention of hepatocellular carcinoma by universal vaccination against hepatitis $B$ virus: the effect and problems. Clin Cancer Res 2005; 11:7953-7957.

57 WHO position paper on Haemophilus influenzae type b conjugate vaccines. Wkly Epidemiol Rec 2006;47:445-452.

58 Akumu AO, English M, Scott AG, Griffiths UK: Economic evaluation of delivering Haemophilus influenzae type $\mathrm{b}$ vaccine in routine immunization services in Kenya. Bull World Health Organ 2007;85:511-518.

55 Pneumococcal conjugate vaccine for childhood immunization - WHO position paper. Wkly Epidemiol Rec 2007;82:93-104.

60 Singh NK: Pneumococcal vaccine concerns: shape-shifters and beyond. Vaccine 2007;25: 5244-5245. 